\title{
ODORES PROVENIENTES DE GRAVES PROBLEMAS AMBIENTAIS
}

\author{
E. M. Sales ${ }^{1}$, J. C. M. dos $\operatorname{Santos}^{1}$ A. B. da $\operatorname{Costa}^{1}$ V. N. F. Lisboa ${ }^{1}$ e A. M de Medeiros ${ }^{2}$ \\ ${ }^{1}$ Universidade Federal de Campina Grande, Departamento de Engenharia \\ ${ }^{2}$ Universidade Estadual da Paraíba, Departamento de Química Industrial \\ E-mail para contato: emanuele.montenegro@gmail.com
}

\begin{abstract}
RESUMO - Um dos problemas mais complexos relacionados à poluição se refere à poluição causada pelos compostos odorantes. Os odores são emanados de inúmeras atividades industriais, tais como refinarias de petróleo e indústrias de cortume. A poluição odorante constitui um grave problema ambiental devido ao seu caráter subjetivo e a falta de leis a nível federal que registrem esse tipo de emissão. Estatísticas revelam que mais de $50 \%$ das reclamações de poluição atmosférica está ligada aos odores. As excessivas emissões odorantes trazem inconvenientes para a saúde da população que variam desde sintomas psicológicos e físicos até problemas respiratórios e desvalorização de imóveis. Dentro dessa linha o presente trabalho aborda uma revisão bibliográfica sobre o tema poluição odorante abrangendo os principais compostos odorantes, problemática dos odores e técnicas de desodorização de efluentes com forte potencial odorante.
\end{abstract}

\section{INTRODUÇÃO}

A poluição atmosférica é tida com um fator preocupante quanto ao meio ambiente e a qualidade de vida. O rápido avanço tecnológico e industrial resultou no aumento das emissões odorantes (CARVALHO et al., 2001). Os odores são inerentes à maioria dos processos industriais e vêm se tornando uma causa de desconforto ambiental para o público (CARMO JÚNIOR et al., 2010), trazendo consigo consequências negativas para a imagem da indústria e para a qualidade de vida da população e do ar (KRUNGER, 2006).

O controle da poluição atmosférica constitui-se como um fator de grande importância na busca da conservação do meio ambiente e na implementação de uma política de desenvolvimento sustentável, uma vez que afeta, de diversas formas, a saúde humana, os ecossistemas e os materiais. A poluição ambiental, por emissões gasosas odoríficas, produzidas pelos diferentes tipos de atividades industriais, converteu-se num problema preocupante, de difícil solução sendo cada vez mais frequentes as queixas e o desconforto ambiental (LISBOA et al. ,2002). A principal preocupação com odor é quanto ao incomodo olfativo que ele causa, mesmo quando presente no ar em concentrações muito baixas. Levantamentos indicam que a maioria das reclamações ambientais sobre poluição na atualidade relativa a odores, explica-se em parte por ser um tipo de poluição com efeitos imediatos sobre as pessoas (SCHWAB, 2003). 


\section{9 a 22 de outubro de 2014 \\ Florianópolis/SC}

Nos últimos 30 anos os odores se tornaram um grave problema ambiental e a discussão sobre os efeitos negativos de odores na saúde humana ainda é uma questão amplamente estudada e debatida (CAPELLI, 2011). O presente trabalho insere-se nos estudos referentes à engenharia ambiental tendo como objeto de estudo a poluição causada por compostos odorantes e toda a problemática referente a odores.

\section{METODOLOGIA}

A metodologia consistiu na realização das atividades de revisão bibliográfica feita mediante referências nacionais e internacionais sobre os temas: poluição odorante, a geração e a problemática do odor advindo do composto odorante abrangendo sua caracterização e efeitos negativos à saúde humana.

\section{REVISÃO BIBLIOGRÁFICA}

\subsection{Odor}

Os odores consistem na disposição de grupos de moléculas, cada uma delas com forma e tamanho definidos. O nariz humano contém mais de 100 milhões de receptores especializados, ou sensores, que atuam conjuntamente em complexas operações para identificação dessas moléculas (FIRESTEIN, 2001; BBC NEWS, 2007 apud LISBOA et al., 2009).Segundo Van Ruth (2001) apud DINCER (2006) o odor é percebido devido a estimulação química de células quimiorreceptora no epitélio olfativo do nariz pelas moléculas gasosas. As moléculas odorantes interagem com receptores no nariz humano e um sinal elétrico é produzido e analisado pelo cérebro a fim de criar uma sensação odorante no indivíduo (HAAS, 2008).

As reclamações do público ao odor dependem da concentração do gás odorante, presença de limiar de percepção, de outros compostos odoríferos, tonicidade e hedonicidade do odor. A presença simultânea de vários gases odorantes na mistura pode criar percepções diferentes em seres humanos em comparação à percepção devido a cada um dos componentes específicos na forma pura na mesma concentração. Assim cada fonte pode ter um caráter de odor diferente e a qualidade do ar criado pela emissão odorante pode ser diferente de uma fonte para outra (DINCER, 2006).

As pessoas expostas a cheiros desagradáveis ficam mais sensível a estes, pessoas que trabalham em áreas que frequentemente contém odores desagradáveis podem se tornar insensíveis aos mesmos. Isto conduz a problemas quando, por exemplo, os responsáveis pela empresa geradora de odores visitam residências onde há queixas sobre odores, resultando em visões amplamente contraditórias sobre o que é um incômodo causado pelo odor (KRUGGER, 2006).

O transporte do odor é afetado por fatores como estações do ano, horário do dia e das condições da atmosfera que influenciam a dispersão do odor (turbulência, velocidade do vento e direção do vento). O potencial de detecção e irritação irá influenciar a resposta por receptores. A percepção do odor pode ser afetada pela combinação de compostos odorantes liberados durante a formação, bem como as características do odorante em si, como duração e frequência da emissão (DREW, 2007). 


\section{9 a 22 de outubro de 2014 \\ Florianópolis/SC}

Os desconfortos causados pelos odores trazem efeitos negativos para a indústria e para a qualidade de vida da população vizinha. $\mathrm{O}$ efeito de um odor é geralmente definido pelos fatores frequência, intensidade, duração e gravidade. A frequência refere-se ao número de vezes que um odor ocorreu, a intensidade refere-se à concentração de um odor, a duração refere-se ao período de tempo em o odor é percebido e a gravidade refere-se ao caráter do odor (quanto agradável ou desagradável ele é, hedonicidade). A intensidade do odor tem recebido a maior atenção por ser considerada a variável principal no controle do odor (RAPPERT e MULLER apud KRUGGER, 2006).

Os efeitos que os odores causam na saúde ainda são difíceis de serem quantificados, entretanto já foram relatados e documentados sintomas de náuseas, vômitos e dor de cabeça; falta de fôlego e tosse; intensificação da asma; distúrbios no sono e no apetite; irritação nos olhos, nariz e garganta; inquietação, tristeza e depressão; redução da sensação de bem-estar e do prazer de realização de atividades cotidianas como comer, passear, etc. Alguns estudos comprovam que a exposição a odores pode causar diferentes efeitos em seres humanos, variando desde tensões emocionais, tais como estados de ansiedade, mal-estar, dor de cabeça, depressão ou a sintomas físicos (CAPELLI, 2011).

Embora os odores desagradáveis causem sintomas em alguns indivíduos, a problemática do odor causada pela poluição do ar é geralmente vista como sendo um incômodo. Normalmente, do ponto de vista da regulamentação ambiental, é visto como um fator da perda do bem-estar e não de prejuízo à saúde (MARIANO, 2001).

Os odores, de maneira geral, sempre fazem parte de uma situação ou processo, provocando as mais diversas reações, tanto em uma única pessoa quanto em toda a população exposta. O maior problema enfrentado, quando se tenta oferecer solução para as reclamações de odor feitas por uma comunidade é a falta de padrões adequados para orientar as autoridades e administradores ambientais das fontes emissoras de tais poluentes (SCHIRMER et al., 2010).

Os odores, segundo McGINLEY, McGINLEY e MANN (2000) correspondem a 70\% das reclamações relacionadas à poluição atmosférica. Portanto é imperativo estudos e pesquisas para avaliar esse tipo de poluição assim como também buscar soluções para essa problemática.

\subsubsection{Legislação Ambiental}

Com relação aos odores no Brasil ainda não há legislação nacional especifica. Cada estado brasileiro tem desenvolvido sua própria legislação para o controle da poluição ambiental (SCHIRMER, 2007).

No estado de São Paulo o Decreto n ${ }^{\circ} 8.468$, de 08 de setembro de 1976, na seção II do artigo 33, proíbe a emissão de compostos odorantes na atmosfera, em quantidades que possam ser perceptíveis fora dos limites da área de propriedade da fonte emissora. Este decreto deu origem à legislação de muitos outros estados (CARMO JÚNIOR, 2005).

Em Santa Catarina, o Decreto $\mathrm{n}^{\circ} 14.250$, de 05 de junho de 1981, regulamenta dispositivos da Lei de 1891, de 15/10/80, referente à proteção e a melhoria da qualidade 
ambiental, proibindo a emissão de substâncias odoríferas na atmosfera em quantidades que possam ser perceptíveis fora dos limites da área de propriedade da fonte emissora e apresenta uma Tabela com 55 substâncias e seus respectivos limites de percepção olfativa (LPO). Nos casos dos compostos que não foram estabelecidos padrão de emissão, deverão ser adotadas medidas de controle de poluição do ar de acordo com a melhor tecnologia disponível (CARMO Jr apud SCHIRMER, 2007).

\subsection{Compostos Odorantes}

\subsubsection{Compostos Sulfurados}

Os compostos sulfurados estão entre as substâncias mais odorantes podendo ser detectadas em baixíssimas concentrações na ordem de ppbs. Quanto à estrutura química se dividem em mercaptanas (-SH) e sulfetos (-S) (BALBINOT).

\subsubsection{Compostos Nitrogenados}

O principal composto odorante nesse grupo é a amônia (BALBINOT, 2010). Assim como a amônia ,as aminas apresentam um odor desagradável aliado a uma alta toxicidade e podem estar relacionadas ao tratamento de águas residuais (biomassa) (SCHIRMER ,2004).

\subsubsection{Moléculas Oxigenadas}

Dentro dessa categoria se tem os aldeídos, álcoois, cetonas, fenóis, hidrocarbonetos (aromáticos), dióxido de enxofre e óxidos de nitrogênio (Adaptado QUADROS, 2004). Como cada substância odorante se diferencia em forma molecular e família, o tipo de resposta que o organismo humano tem também varia de acordo com o tipo de composto presente no ar. Esta resposta pode ser uma reação - de agrado, desagrado ou indiferença - que o indivíduo pode ter em relação ao composto ou até mesmo a sua percepção em si. Dessa forma os efeitos para a saúde de grandes exposições a substâncias odorantes também variam de acordo com o tipo de substância. Como os compostos odorantes são naturalmente inalados, estes podem ser absorvidos pela corrente sanguínea e causar diversos males dependendo da sua origem (QUADROS, 2004).

\subsection{Origem dos Compostos Odorantes}

Os compostos odorantes se originam de várias fontes ou atividades tais como atividades agroindustriais (lacticínios, indústrias de processamento de alimentos e bebidas), industriais (revestimentos, pasta de papel, etc.), agropecuárias, tratamento de resíduos e de efluentes líquidos e gasosos (PINHO, 2008).

A maior parte dos odores gerados no processo industrial é originada da digestão anaeróbia da matéria orgânica. É grande o número de compostos odorantes gerados os quais podem ser classificados em compostos sulfurados, compostos nitrogenados e moléculas oxigenadas ou 
aromáticas (ácidos graxos voláteis, cetonas, aldeídos, etc.) (GELINAS e HEROUX, 2004 apud BALBINOT,2010).

Estudos indicam que há algumas características comuns aos compostos odorantes: hidro solubilidade (facilidade em solubilizar-se na água), boa pressão de vapor (tende a evaporar com facilidade), lipofilicidade (capacidade de dissolver-se em gorduras) e baixa massa molar (fator relacionado com baixa densidade e alta volatilidade) (SCHWAB, 2003).

\subsection{Técnicas dos Compostos Odorantes}

Dentre as técnicas de análises de odor serão abordadas as Técnicas Sensoriais ou Olfatométricas, os Métodos físico-químicos e o Nariz eletrônico.

\subsubsection{Técnicas Sensoriais ou Olfatometria}

Essa técnica consiste na utilização do nariz humano como instrumento, se baseando em diluições de uma substância padrão para determinar faixas de intensidade de odores. Para aplicar essa técnica se pode fazer uso de aplicação de questionários, método do butanol e uso do olfatômetro (QUADROS, 2004).

A aplicação de questionários é feita a partir de uma seleção de pessoas capazes de responder questões relacionadas a odores, tais como, para saber se odor é agradável ou não, com que frequência ocorre a sensação de odor, entre outras. Com relação ao método do butanol a metodologia consiste em diluir as amostras referenciais e comparar com uma amostra padrão o qual a resposta a ser analisado será para classificar o cheiro de acordo com uma escala de intensidade de odor definida por normas e isso será feito por um grupo de pessoas previamente selecionadas (QUADROS, 2004).

O uso do olfatômetro de diluição serve para determinar a concentração do odor de uma fonte sendo necessário um júri de pessoas preparadas para estas medições. O parâmetro concentração do odor de uma amostra é expresso em UO.m ${ }^{-3}$ (unidade de odor/metro cúbico de ar) (STUETZ e FRENCHEN et al, 2001 apud BELLI FILHO et al., 2007).

A olfatometria objetiva avaliar incômodos, determinar os riscos e os limites de exposição dos odores sobre as pessoas. Os odores podem ser avaliados por meio de enquetes olfatométricas realizadas junto às comunidades, tendo-se como modelo as recomendações da norma VDI 3883 (1993) para a determinação da percepção humana sobre o seu ambiente. Para a avaliação da intensidade de um odor existem escalas de referências que representam graus diferentes de incômodos, podendo seguir recomendações normatizadas. Para a avaliação desta variável pode-se trabalhar com pessoas treinadas ou diretamente com cidadãos de um local a ser avaliado (BELLI FILHO et al., 2007)

A olfatometria fornece uma compreensão global do odor, haja vista permitir verificar a sensibilidade do sistema olfativo humano, que frequentemente percebe sinais químicos correspondentes a pequenas concentrações moleculares não detectados facilmente pelos analisadores 
químicos. Essa técnica também permite a integração das propriedades da membrana da mucosa nasal com a mistura de moléculas odorantes. De fato, quando a membrana da mucosa estiver sujeita a uma mistura de moléculas, pode haver uma inibição ou uma estimulação resultante dos sinais do odor que seriam obtidos das moléculas examinadas separadamente (GOURONNEC e TOMASSO, 2000 apud VIEIRA, 2007).

\subsubsection{Métodos Físico-Químicos}

Os métodos físico-químicos buscam identificar e quantificar, com auxílio de instrumentos analíticos, cada um dos compostos odorantes presentes no efluente gasoso. Entre estas técnicas encontram-se as análises volumétricas, colorimétricas, a cromatografia gasosa, a espectrometria de massa, e outras (MICHEL, 1997 apud VIEIRA, 2007).

As análises físico-químicas têm por finalidade detectar a presença de compostos, odorantes ou não, numa amostra de gás ou ar atmosférico e de ambientes fechados. Conforme o que foi observado em diversos artigos, a Cromatografia Gasosa parece ser a técnica mais utilizada atualmente, além das técnicas de absorção química, para a detecção de determinados compostos gasosos, sendo que esta ainda pode ser acoplada a uma unidade de dessorção térmica e outra de espectrometria de massa (QUADROS, 2004).

\subsubsection{Nariz Eletrônico}

Narizes Eletrônicos têm sido desenvolvidos para detecção automática e classificação de odores, vapores e gases. Um Nariz Eletrônico é geralmente composto por um sistema de sensores químicos e um sistema eletrônico associados à inteligência artificial, para reconhecimento (PIOGGIA, 2007 apud LISBOA et al., 2009).

O funcionamento do nariz eletrônico consiste na utilização de um conjunto de captores não seletivos que reagem na presença de compostos voláteis, formando assim uma impressão digital da mistura odorante em estudo. O papel do sistema de reconhecimento é associar esta marca eletrônica a um determinado odor, após um tratamento matemático previamente implementado sobre um microprocessador. Sendo assim, de acordo com suas aplicações, é possível identificar, detector, ou ainda, discriminar diferentes odores (HUDON, 1999 apud VIEIRA, 2007).

\section{Métodos Físico-Químicos}

Este trabalho objetivou fazer um breve referencial teórico a cerca do tema poluição atmosférica abrangendo o conceito de odor, os compostos odorantes, legislação ambiental no Brasil sobre compostos odorantes e as principais técnicas de mensuração do odor. Assim se pode perceber que a poluição causada por compostos odorantes constitui uma problemática que afeta tanto a saúde humana como a qualidade do ar, sendo necessário um controle mais rígido pelos órgãos ambientais e pela criação de normas de âmbito nacional para o controle de odor. Com relação às técnicas de tratamentos não existe um método padrão para tratar odores, ficando a critério de quem for realizar o tratamento escolher qual melhor técnica é eficaz para o tratamento. 


\section{Referências}

BALBINOT, A. Poluição olfativa-Composição, mensuração e técnicas de tratamento de efluentes com potencial odorífero. Trabalho de Término de curso (Bacharelado em Engenharia Química). Universidade Federal do Rio Grande do Sul, Porto Alegre, 2010.

BELLI FILHO, P; PIHEIRO DA SILVA, G; SANTO, C. DE L. LISBOA, H. DE M. CARMO JÚNIOR, G. N. DO. Avaliação de impactos de odores em bacias hidrográficas com produções de suínos. Revista Engenharia Sanitária e Ambiental, v.12 n³, jul/set 2007.p.252-258.

CAPELLI,L.;SIRONI,S.; ROSSO,R.D;CENTOLA,P.; ROSSI, A. Odour impact assessment in urban areas: case study of the city of Terni. Procedia Environmental Sciences, nº,p.151157,2011 .

CARMO JÚNIOR, G.R da R. Otimização e Aplicação de metodologias para análises olfatométricas integradas ao saneamento ambiental. Tese (Doutorado em Engenharia Ambiental). Pós-graduação em Engenharia Sanitária e Ambiental. Universidade Federal de Santa Catarina, Florianópolis 2005.

CARVALHO,C.M.de;WOLFF,D.B.;RIBEIRO,L.G.;CHIARANDA,H.L.COSTA,R.H.R.da;BEL LI FILHO,P. Biofiltração para desodorização em reatores anaeróbios. XXI Congresso Brasileiro de Engenharia Sanitária e Ambiental,2001.

DINCER,F.; MUEZZNOGLU, A. Chemical characterization of odors due to some industrial and urban facilities in Izmir, Turkey. Atmospheric Environment, n 40, P.4210-4219,2006

DREW,G.H.,SMITH,R.,GERARD,V.BURGE,C.LOWE,M.KINNERSLEY,R.SNEATH.R,LON GHURST.Approateness of selecting different averaging times for modeling chronic and acute exposure to environmental odours. Revista Atmospheric Environment, ${ }^{\circ}$ 41, ano 2007,pg 28702880

HAAS, T.; LAMMERS, P. S. ;DIEKMANN, B.; HORNER, G.; BOECKER, P. A method for online measurement of odour with a chemosensor system. Sensors and Actuators B, $\mathrm{n}^{\circ} 132 \mathrm{p}$. 545-550,2008

LISBOA, H.de M. PAGE, T. GUY, C. Gestão de odores: Fundamentos do nariz eletrônico. Revista Engenharia Sanitária e Ambiental, v.14,n¹,p.9-18,jan/mar 2009

KRUGER,R. L. Identificação e degradação de compostos odoríferos originados no processamento de aves. Dissertação (Mestrado em Engenharia de Alimentos). Programa de Mestrado em Engenharia de Alimentos. Universidade Integrada do Alto Uruguai e das Missões. Erechim, Rio Grande do Sul, Brasil,2006.

MARIANO, J. B. Impacto do refino do petróleo. Tese (Mestrado em Ciências em Planejamento energético). Pós-graduação em Egenharia. Universidade Federal do Rio de Janeiro, 2001.

McGINLEY, C. M.; McGINLEY, M. A.; MANN, J. Olfactomatics: applied mathematics for odor testing. In.: WATER ENVIRONMENT FEDERATION ODOR - VOC 2000 SPECIALTY CONFERENCE. Cincinnati. 2000. Disponível em CD-ROW. 
PINHO, A. C. M. de. Desenvolvimento de um olfatômetro dinâmico. Dissertação (Mestrado em Engenharia Ambiental). Universidade de Aveiro, 2008.

QUADROS, M. E. Avaliação de odores em aterros sanitários e certificação de um júri à olfatometria. Trabalho de término de conclusão de curso de Engenharia Sanitária e Ambiental na Universidade Federal de Santa Catarina, Florianópolis, Santa Catarina, 2004.

SCHAWB, F.C.C. Odores incômodos em emissões industriais: aspectos teóricos, práticas atuais e um estudo de caso em fábrica agroquímica. Dissertação (Mestrado em Engenharia Sanitária e Ambiental). Universidade do Rio de Janeiro, Rio de Janeiro, 2003.

SCHIRMER, W. N. Amostragem, análise e proposta de tratamento de compostos orgânicos voláteis $(\mathrm{COV})$ e odorantes em estação de despejos industriais de refinaria de petróleo. Dissertação (Mestrado em Engenharia Ambiental)-Pós-Graduação em Engenharia Sanitária Ambiental, Universidade Federal de Santa Catarina, Florianópolis, 2004.

SCHIRMER, W. N. Avaliação do desempenho de nanotubos de carbono "cup-stacked" (cscnt) na remoção de compostos orgânicos voláteis (cov) decorrentes gasosas. Trabalho de Qualificação de Doutorado. Programa de Pós-graduação em Engenharia Ambiental. Universidade Federal de Santa Catarina, Florianópolis, Santa Catarina, 2007.

SCHIRMER, W. N.; OLIVEIRA, G. L. de. Desodorização de efluentes líquidos tratados em leito de zona de raízes ("wetlands"). Revista TECNO-LÓGICA, Santa Cruz do Sul, v. 14, n’1, p. 1119, jan/jun. 2010.

VIEIRA, M. M. Avaliação da eficiência de um lavador de gases no tratamento dos efluentes gasosos de um restaurante no município de Florianópolis. Trabalho de Término de conclusão do curso (Graduação em Engenharia Sanitária e Ambiental). Universidade Federal de Santa Catarina, Florianópolis, 2007. 